\title{
Dynamic orientation of a deuteron-spin system by rf irradiation of a polarized-proton-spin system
}

\author{
W. de Boer* \\ CERN, Geneva, Switzerland \\ (Received 3 February 1975)
}

\begin{abstract}
We have observed a high degree of orientation of a deuteron-spin system in partially deuterated organic materials. This was obtained by dynamic polarization, using a $25-\mathrm{kG}$ magnetic field and a dilution refrigerator, followed by slightly off-resonance rf irradiation of the polarized-proton-spin system, which causes a cooling of the proton spin-spin interaction reservoir. Thermal contact between this reservoir and the one made up of the deuteron quadrupole interactions provides a simultaneous cooling of the latter reservoir, which corresponds to a change in the deuteron tensor polarization or alignment. Alignment values around $60 \%$ were observed for some parts of the inhomogeneous deuteron-spin system, which corresponds to a spin termperature for the quadrupole reservoir of $7 \mu \mathrm{K}$. The dependence of the alignment on radio frequency and initial proton polarization as well as the thermal mixing rates are in good agreement with quantitative estimates from the spin-temperature theory.
\end{abstract}

\section{INTRODUCTION}

We report here dynamic orientation experiments on partially deuterated organic materials, doped with paramagnetic $\mathrm{Cr}^{5+}$ complexes. The magnetic properties of such samples have attracted much attention, because very high vector polarizations can be obtained in them: polarizations above $95 \%$ for protons, ${ }^{1} 40 \%$ for deuterons, and $50 \%$ for ${ }^{13} \mathrm{C}$ nuclei have been reported. ${ }^{2,3}$ This has found applications in high-energy scattering experiments, where these materials are used as polarized target material.

Such high polarizations are obtained by irradiating the electron-spin system near the magnetic resonance frequency of the $\mathrm{Cr}^{5+}$ complexes (69.5 $\mathrm{GHz}$ in a $25-\mathrm{kG}$ magnetic field). This causes a cooling of the "electron spin-spin interaction reservoir." The concept of a spin-spin interaction reservoir, representing the interaction energy amongst spins, was introduced by Provotorov, ${ }^{4}$ who generalized Redfield's spin-temperature hypothesis. ${ }^{5}$ This concept appeared to be extremely useful for the understanding of the behavior of a spin system in a rigid lattice. It led also to the prediction of several new phenomena, which were experimentally verified first for homogeneous spin systems (Abragam, ${ }^{6}$ Goldman, ${ }^{7}$ Anderson and Hartmann, ${ }^{8}$ Philippot, ${ }^{9}$ Jeener, ${ }^{10}$ and Hebel ${ }^{11}$ ) and later for inhomogeneous spin systems as well (Atsarkin and Rodak, ${ }^{12}$ Wenckebach and Poulis, ${ }^{13}$ and de Boer, Borghini, Morimoto, Niinikoski, and $\mathrm{Udo}^{2}$ ).

Thermal mixing between the electron spin-spin interaction reservoir and the nuclear Zeeman reservoirs causes simultaneous polarization of all nuclei contained in the sample (see review by Borghini $\left.{ }^{14}\right)$.
An analogous mechanism of dynamic orientation occurs in partially deuterated materials if the proton-spin system in such samples is irradiated with a slightly off-resonance rf field, which causes a cooling of the proton spin-spin interaction reservoir. It appears that there exists a reasonably strong thermal mixing between this reservoir and the one made up of the deuteron quadrupole and dipolar interaction energy, even in a $25-\mathrm{kG}$ magnetic field. This latter reservoir then becomes cooled as well during rf irradiation of the protonspin system. A similar thermal contact between the spin-spin interaction reservoirs of different nuclear spin species in a high-magnetic field was observed by Zhabotinskii et al. ${ }^{15}$ for homogeneous spin systems. As will be shown below, cooling of the deuteron quadrupole reservoir can result in a pure deuteron tensor polarization or alignment. Such a pure deuteron tensor polarization, obtained by irradiation of a polarized proton spin system, was first observed ${ }^{16}$ in ethanediol-D4 $\left[\mathrm{C}_{2} \mathrm{D}_{4}(\mathrm{OH})_{2}\right]$.

In Sec. III we will compare various basic aspects of these experiments, such as the behavior of a spin system under irradiation, the thermal contact between different nuclear-spin species in a high-magnetic field, their interaction with an electron spin-spin interaction reservoir, and relaxation phenomena, with the spin-temperature theory, which has been so successful in describing such a wide variety of phenomena.

The initial spin temperature of a proton-spin system can be made low in absolute value by dynamic polarization. After the initial step of dynamic polarization, the second step, consisting of rf irradiation of the polarized proton-spin system, makes it possible to obtain spin temperatures in the $\mu \mathrm{K}$ region.

Unfortunately, the presence of paramagnetic im- 
purities, necessary for dynamic polarization, shortens the nuclear-spin-lattice relaxation times, thus reducing the maximum alignment obtainable. However, by using temperatures around $0.1 \mathrm{~K}$, which can be obtained with a ${ }^{3} \mathrm{He}-{ }^{4} \mathrm{He}$ dilution refrigerator, this disadvantage can be eliminated quite effectively.

\section{THEORY}

\section{A. Introduction}

The orientation of a system of spins $I$ along an axis $\vec{z}$ can be described by so-called orientation parameters, which are defined as expectation values of irreducible spin tensors. ${ }^{17}$ Here we will consider only the vector polarization $P$, which is defined as

$$
P=\left\langle I_{z}\right\rangle / I
$$

and the tensor polarization or alignment $A$, defined as

$$
A=\left\langle 3 I_{z}^{2}-I(I+1)\right\rangle /[I(2 I-1)] .
$$

The orientation parameters of higher order in $I_{k}$ are normally null or very small, and can be neglected in practice. For spin $I=\frac{1}{2}$, the alignment is always zero and the orientation coincides with the (vector) polarization.

If a spin $I$ is subjected to a magnetic field $H$ in the direction $\vec{z}$, then the Zeeman interaction $\mathfrak{F}_{Z I}$ $=-\vec{\mu} \cdot \overrightarrow{\mathrm{H}}$ establishes a set of $2 I+1$ sublevels with energy $E_{m}=-m \hbar \gamma H ; \mu=|\gamma| \hbar I=g \mu_{B} I$ is the magnetic dipole moment, $|\gamma|$ is the gyromagnetic ratio, $\hbar$ is Planck's constant, divided by $2 \pi$, the symbol $m=\left\langle I_{z}\right\rangle, g$ is the spectroscopic splitting factor, and $\mu_{B}$ is the Bohr magneton.

The polarization of an assembly of identical nuclei with $I=\frac{1}{2}$ is calculated from Eq. (1) to be

$$
P=n_{+}-n_{-},
$$

where $n_{*}$ is the fraction of the spins in the $m=+\frac{1}{2}$ state, and $n_{-}$is the fraction with $m=-\frac{1}{2}$.

If the spins are in thermal equilibrium, they can be characterized by a temperature $T_{S}$. The distribution of the different spins over the magnetic sublevels is then given by the Boltzmann law,

$$
n_{-} / n_{+}=\exp \left[\left(E_{+}-E_{-}\right) / k T_{S}\right] \text {. }
$$

From Eqs. (3) and (4), the polarization for $I=\frac{1}{2}$ is found to be

$$
P=\tanh \left(\mu H / k T_{S}\right),
$$

which is a special case of the general Brillouin formula. At sufficiently high temperatures, Eq. (5) can be approximated by

$$
P=\mu H / k T_{S}=\frac{1}{2} h \nu / k T_{S}
$$

where $\nu=g \mu_{B} H / h$ is the Larmor frequency of the spin system, and $h=$ Planck's constant.

\section{B. Description of the deuteron-spin system}

Let us briefly recall some properties of the deuteron-spin system. ${ }^{18}$ In an external magnetic field, the energies of the three magnetic substates can be written as

$E_{m}=-h \nu_{D} m+h \nu_{Q}\left[\left(3 \cos ^{2} \theta-1\right)\right]\left[3 m^{2}-I(I+1)\right]$,

where $\nu_{D}$ is the deuteron Larmor frequency (16.34 $\mathrm{MHz}$ in a $25-\mathrm{kG}$ magnetic field), $m=\left\langle I_{z}\right\rangle$, and $\nu_{Q}$ $=e^{2} q Q / 8 h=+19.5 \mathrm{kHz}$ for propanediol-D6, where $e q$ is the deuteron quadrupole moment, and $e Q$ is the value of the electrical-field gradient along the principal axis of the field-gradient tensor in case of axial symmetry. This axis can be assumed to coincide with a $C-D$ bond; $\theta$ is the angle between it and the direction of the magnetic field. In disoriented solids, as is our case, all orientations of $\mathrm{C}-\mathrm{D}$ bonds are equally probable. Therefore the magnetic levels are shifted, depending on the value of $\theta$. This gives rise to two transitions, which vary in energy according to the factor $3 \cos ^{2} \theta-1$, and in intensity according to the factor $1 / \cos \theta$ since the intensity is proportional to

$$
\frac{d n_{D}}{d \nu_{D}} \propto \frac{\sin \theta d \theta}{d\left(3 \cos ^{2} \theta-1\right)} \propto \frac{1}{\cos \theta} .
$$

$d n_{D}$ is the number of deuterons, for which the direction of the $C-D$ bond varies between $\theta$ and $\theta+d \theta$. These two transitions partially overlap and the actual deuteron-magnetic-resonance (DMR) line is a superposition of them, as shown in Fig. 1. The two peaks correspond to $\theta=90^{\circ}$, the pedestals to $\theta=0^{\circ}$, while the right-hand peak and lefthand pedestal correspond to the $m=1$ to $m=0$ transition, whose intensity is $\mathrm{J}^{+}$, and the other peak and pedestal to the $m=0$ to $m=-1$ transition with intensity $J^{-}$. The intensities are proportional to the difference in populations $n_{m}$ of the corresponding states, which satisfy the Boltzmann relations under thermal equilibrium conditions. We assume the sum of $n_{m}$ to be normalized to 1 . The vector polarization $P_{D}=\left\langle I_{g}\right\rangle$ is then given by $n_{+1}$ $-n_{-1}=c\left(J^{+}+J^{-}\right)$, and the tensor polarization or alignment $A_{D}=\left\langle 3 I_{z}^{2}-I(I+1)\right\rangle$ is equal to $1-3 n_{0}$ $=c\left(J^{+}-J^{-}\right)$. From the definitions it follows that under thermal equilibrium, $A_{D}$ and $P_{D}$ are related by $A_{D} \cong \frac{3}{4} P_{D}^{2}$, if $A_{D}$ is small. The constant $c$ can be determined by measuring the thermal equilibrium signal at a known temperature. At high polarizations, the intensities $J^{*}$ and $J^{-}$become different, and the asymmetry, defined as $R=J^{*} / J^{-}$, is related to $P_{D}$ by

$$
\left|P_{D}\right|=\left(R^{2}-1\right) /\left(R^{2}+R+1\right) \text {. }
$$

A comparison of Eqs. (2) and (7) shows that the 
(a)
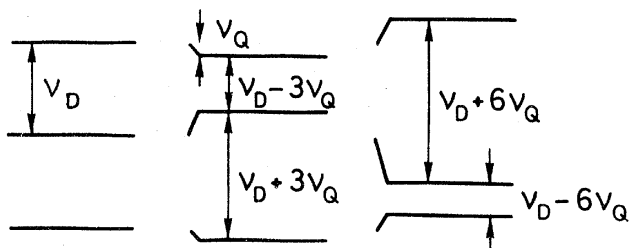

$\Theta=\arccos (\sqrt{1 / 3}) \quad \Theta=\pi / 2$

$\Theta=0$

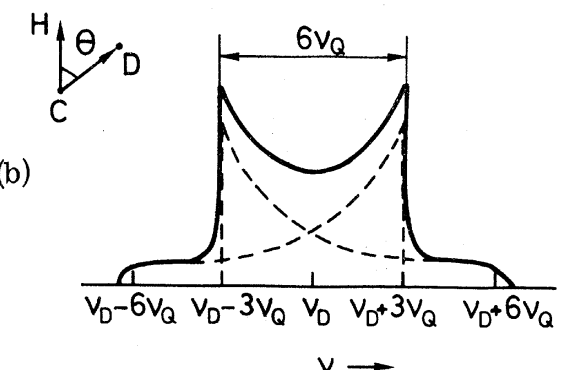

(b)

$\checkmark \rightarrow$

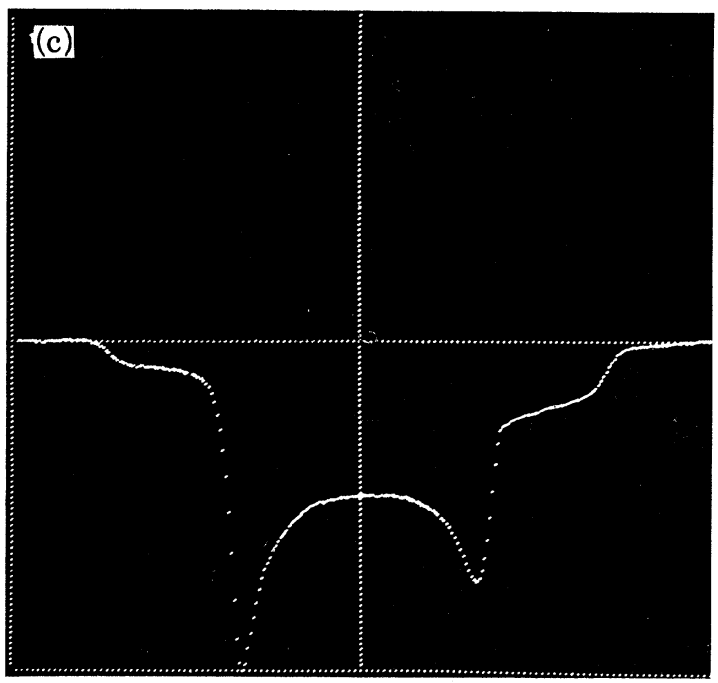

FIG. 1. (a) Energy-level diagram of the deuteron-spin system for three values of the factor $3 \cos ^{2} \theta-1$, namely, $0,-1$, and 2 , which correspond to the center, the peaks, and the pedestals of the deuteron line, respective1y. $\theta$ is roughly the angle between the direction of a C-D bond and an external magnetic field. (b) Theoretical deuteron line shape, which is the sum of the two possible transitions $m=0$ to $m=1$ and $m=-1$ to $m=0$ (dashed lines). Some line broadening due to spin-spin interactions has been taken into acount, which makes the line smoother. Otherwise the peaks would tend to infinity。 (c) Deuteron-magnetic-resonance line in propanediol-D6, measured in a 25-kG magnetic field. The distance between the peaks is $117 \mathrm{kHz}\left(=6 \nu_{Q}\right)$. The deuterons are dynamically polarized to $-43 \%$, which causes the asymmetry [see Eq. (9)].

deuteron quadrupole energy is proportional to the alignment.

\section{Spin-temperature theory}

Following Provotorov ${ }^{4}$ a spin system in an external magnetic field can be characterized by two spin temperatures, one for the Boltzmann distribution between the energy levels having different Zeeman energies which we call $T_{z}$, and one-called $T_{S \text { S }}$-for the Boltzmann distribution between the levels which have approximately the same Zeeman energy, but which may have slightly different energies owing to broadening by spin-spin interactions. $T_{z}$ and $T_{s s}$ are equal to each other, if the spin system is in thermal equilibrium with the lattice. However, this need not be the case. They become different, for example, during slightly off-resonance irradiation of the spin system with an oscillating magnetic field ( $\mathrm{rf}$ or microwave). ${ }^{4,7}$ The evolution of $T_{z}$ and $T_{S S}$ is then governed by the Provotorov equations, which show that after strong irradiation (strong in comparison with the spin-lattice interaction)

$$
T_{S S}=\frac{\Delta}{\bar{\nu}} T_{Z}
$$

where $\bar{\nu}$ is the average Larmor frequency, and $\Delta$ is the difference between $\bar{\nu}$ and the irradiation frequency $\nu$. Thus the absolute value of $T_{S s}$ becomes much smaller than $T_{z}$. This can be understood as follows: Since the irradiation of the spin system occurs off-resonance, there is a difference between the energy absorbed from the oscillating magnetic field and the change in Zeeman energy. This difference is balanced by a change in the spin-spin interaction energy, ${ }^{4,10}$ thus changing $T_{S S}$. Such a change in the spin-spin interaction energy can be thought of as a quick rearrangement between the spins via flip-flop transitions, called cross relaxation transitions. Cross relaxation is assumed to be much faster than spin-lattice relaxation, so the spin system can always stay in internal thermal equilibrium. Under those conditions, the spin-temperature theory is not restricted to homogeneous spin systems, but may be applied to inhomogeneous spin systems as well. ${ }^{8,12-14}$ Most of the experimental verifications of the spin-temperature theory were done under conditions where the so-called high-temperature approximation is valid, which means that higher-order terms in the series expansion of the density matrix can be neglected. This is only allowed at magnetic field values and temperatures where the vector polarization of the spin system under consideration is much smaller than one. If this approximation cannot be made, the expressions become more complicated.

The initial proton-polarization value in our experiments is about 0.9 , so we cannot use the hightemperature approximation to calculate the initial energy. However, the final state is such that the high-temperature approximation proves to be not too bad, as will be discussed later on.

We assume that our system of protons and deuterons in a high external magnetic field can be de- 
scribed by the following density matrix:

$$
\rho=\frac{\exp \left(-\alpha \mathcal{H}_{Z H}-\beta \mathcal{H}_{\text {Int }}-\delta \mathcal{H}_{Z D}\right)}{\operatorname{Tr}\left[\exp \left(-\alpha \mathcal{H}_{Z H}-\beta \mathcal{H}_{1 \mathrm{nt}}-\delta \mathcal{K}_{Z D}\right)\right]},
$$

where $\mathfrak{H}_{Z H}$ and $\mathfrak{H}_{Z D}$ are the Hamiltonians for the proton and deuteron Zeeman energies, while $\mathcal{H}_{\text {int }}$ includes the dipolar energy amongst all spins plus the deuteron-quadrupole interaction energy. Furthermore, $\alpha=h / k T_{Z H}, \beta=h / k T_{1 \mathrm{nt}}$, and $\delta=h / k T_{Z D}$, where $T_{Z H}$ and $T_{Z D}$ are the temperatures of the proton and deuteron Zeeman reservoirs, respectively, and $T_{\mathrm{int}}$ is the temperature characterizing $\mathfrak{H}_{\text {int }}$.

The different nuclear-spin species are supposed to have a common $T_{\text {int }}$. Such an assumption was first made and experimentally verified for homogeneous nuclear-spin systems. ${ }^{15}$ For inhomogeneous spin systems, as is the case for deuterons, such an assumption will only be valid if the mechanisms for establishing a common $T_{1 \mathrm{nt}}$ within the reservoir corresponding to the energy $\left\langle\mathcal{H}_{\text {int }}\right\rangle$ are strong enough to obtain always an equilibrium situation within this reservoir. The reservoir is called the quadrupole interaction reservoir, since a large contribution to $\left\langle\mathcal{F}_{\mathrm{int}}\right\rangle$ comes from the deuteron quadrupole energy. Here we tacitly assumed that the nonsecular part of $\mathcal{H}_{1 \text { nt }}$ is negligible, which is true in a high external magnetic field. If this is not the case, one is not allowed to speak about separated subsystems of a spin system, because the nonsecular terms bring them rapidly into equilibrium with each other. ${ }^{7}$ Thermal equilibrium may be established in the quadrupole reservoir by two mechanisms, namely via cross relaxation between various spins and via "forbidden" transitions induced by the rf field, for example one proton flips simultaneously with two deuteron spins, which flip in opposite directions. ${ }^{16}$ The latter mechanism is probably less effective, since it involves at least three spins which flip simultaneously. However, it need not be negligible for deuterons, which belong to the pedestals of the DMR line, because their low concentration makes the first mechanism rather slow. We will return to this point in Sec. III.

In our experiments, we reduced the deuteron vector-polarization to zero by application of a resonant saturating rf field; hence $\delta=0$, which simplifies the analysis of the results. After this step, the still-polarized proton-spin system was irradiated, thus changing the value of $\beta$. Since the spinlattice relaxation times are normally much longer than the time span for these experiments, we can neglect them in the following calculations and eventually make a correction in the experimental results. The value of $\beta$ can be calculated from energy conservation, which leads to

$$
-\frac{1}{2} h \Delta\left(P_{H i}-P_{H f}\right) n_{H}=-\frac{1}{4} h \beta_{f} \Omega^{2} n_{H},
$$

where $i$ and $f$ denote the initial and final states, respectively. $P_{H}$ is the proton polarization, and $n_{H}$ is the number of protons in the sample; the symbol $\Omega^{2}$ is defined by the relation

$$
-\frac{1}{4} h \beta_{f} \Omega^{2} n_{H}=\operatorname{Tr}\left(\rho \mathcal{H}_{1 \mathrm{nt}}\right) \text {. }
$$

The left-hand side of Eq. (12) is the difference in proton Zeeman energy between the initial and final state, multiplied by $\Delta / \nu_{H}$, which then gives the amount of energy taken up by the quadrupole reservoir, since for each absorbed photon an amount $\left|h \nu_{H}-h \nu\right|=h \Delta$ is taken from the quadrupole reservoir to balance the energy. In Eq. (13), which gives the energy of the quadrupole reservoir in the final state, we have neglected higher-order terms of $\beta$ involved in the series expansion of $\rho$, which then takes the form

$$
\rho^{\prime}=\frac{1}{\Sigma} \exp \left(-\alpha \mathcal{H}_{Z H}\right)\left(1-\beta \mathcal{H}_{\text {int }}\right),
$$

where $\Sigma$ is a normalization constant. The errors introduced by this approximation will be discussed later. On the right-hand side of Eq. (12), we also neglected the initial energy of the quadrupole reservoir, since $\left|\beta_{i}\right|$ is normally much smaller than $\left|\beta_{f}\right|$ under our experimental conditions.

After rf irradiation, $P_{H f}$ is normally so small that we can write it as [see Eq. (6)]

$$
P_{H f}=\frac{1}{2} \alpha_{f} \nu_{H}=\frac{1}{2} \beta_{f} \Delta,
$$

where $\nu_{H}$ is the proton Larmor frequency. The right-hand side of Eq. (15) has been obtained by using Eq. (10).

Substitution in Eq. (12) gives

$$
\beta_{f}=2 \Delta P_{H i} /\left(\Omega^{2}+\Delta^{2}\right) \text {. }
$$

From this equation, it follows that the maximum value of $\beta_{f}$ occurs at a radio frequency for which $|\Delta|=\Omega$; it is then equal to $P_{H i} / \Omega$.

Equations (15) and (16) combined yield

$$
P_{H f}=\Delta^{2} P_{H i} /\left(\Omega^{2}+\Delta^{2}\right) \text {. }
$$

We now try to estimate the errors introduced by using $\rho^{\prime}$ instead of $\rho$. Since deuterons are three times more abundant than protons in our sample, and since the quadrupolar broadening of the deuteron spin system is large compared with the dipolar broadening, we approximate $\left\langle\mathcal{H C}_{\text {int }}\right\rangle$ by the quadrupole energy, which is independent of the Zeeman energies, since we made the deuteron polarization zero. Then the quadrupole energy is $h X A_{D}$, where $X=\nu_{Q}\left(3 \cos ^{2} \theta-1\right)$; the alignment $A_{D}$ is defined by

$$
A_{D}=\operatorname{Tr} \rho\left[3 I_{z}^{2}-I(I+1)\right],
$$

which can be reduced to

$$
A_{D}=\left(2 e^{-\beta X}-2 e^{2 B X}\right) /\left(2 e^{-\beta X}+e^{2 B X}\right) \text {. }
$$


If one compares this value of $A_{D}$ with the value obtained by using $\rho^{\prime}$ instead of $\rho$, which yields $A_{D}$ $=-2 \beta X$, it appears that for our range of $\beta$ 's the relative differences are of the order of the experimental errors, which are $10 \%-20 \%$. The main reason why $\beta_{f}$ is still small enough to allow the high-temperature approximation, in spite of the fact that the value of the initial proton polarization is normally 0.9 , is that only half of the proton Zeeman energy is used for cooling of the quadrupole reservoir, as follows from $\mathrm{Eq}$. (17), if one notes that the maximum value of $\beta_{f}$ occurs at $|\Delta|=\Omega$. A larger value of $\beta_{f}$ can be obtained if one uses all the available Zeeman order by performing, after dynamic polarization, an adiabatic fast passage for both proton and deuteron spins. ${ }^{15}$ Besides the above-mentioned approximations, we also considered the proton system as being an homogeneous spin system by assuming in Eq. (12) that all protons have the same Larmor frequency, which is not exactly true because of the inhomogeneous broadening owing to the presence of paramagnetic impurities. Instead of using one $\Delta$ in Eq. (12), one should rather make a summation over all protons, each having its own Larmor frequency and polarization. Furthermore, it is difficult to calculate the value of $\Omega^{2}$ exactly in a noncrystalline material with paramagnetic impurities. Therefore, we will determine its value experimentally by fitting a curve, as derived from Eq. (17), through the experimental points.

From the Provotorov equations it can be derived that the evolution of $\alpha$ and $\beta$ is exponential, with a time constant given by ${ }^{4,7}$

$$
\tau_{\mathrm{mix}}^{-1}=W\left(1+\Delta^{2} / \Omega^{2}\right),
$$

where $W$ is the transition probability for a proton spin under influence of the rf field, which is proportional to the square of the rf field strength and the line-shape function $h(\nu)$ of the proton spin system.

\section{Spin-lattice relaxation}

It was shown by Bloembergen ${ }^{19}$ that the main cause for nuclear spin lattice relaxation in solids with paramagnetic impurities is the fluctuating mag. netic field at the nucleus created by the transitions within the electron spin system. At temperatures where the electron-spin system is almost completely polarized, there are very few electronspin transitions, and consequently, very long nuclear-spin-lattice relaxation times are then observed. ${ }^{1,20,21}$ Furthermore, the various nuclearspin systems are coupled to the electron spin-spin interaction reservoir. ${ }^{2,12-14,21}$ This coupling, which depends on temperature and the presence of microwave power, ${ }^{21}$ tends to equalize the spin temperatures of different nuclear-spin species.
We measured the various time constants for such processes. $^{2}$ It appears that the deuteron quadrupole energy decays with a time constant $T_{A_{D}}$, which is two orders of magnitude shorter than the spinlattice relaxation time of the nuclear Zeeman energy. This time constant shows a strong temperature dependence, $2 \times 10^{4} \mathrm{sec}$ at $0.1 \mathrm{~K}, 2 \times 10^{3} \mathrm{sec}$ at $0.25 \mathrm{~K}$, and $200 \mathrm{sec}$ at $0.4 \mathrm{~K}$.

This temperature dependence is similar to that of the spin-lattice relaxation times for protons and deuterons, denoted by $T_{1 H}$ and $T_{1 D}$, respectively. ${ }^{2}$ Therefore, the quick decay of the alignment is likely to proceed via the electron spin-spin interaction reservoir. The quadrupole energy can be transferred to this reservoir via cross-relaxation transitions in which electron spins and nuclear spins undergo flip-flop transitions simultaneously.

The steep temperature dependence arises then from the fact that at low temperatures, the electron spin system becomes nearly $100 \%$ polarized; that is, all electron spins go to the lowest-energy state. Hence the cross-relaxation rate becomes very slow, because a combination of a "spin-up" and "spin-down" is a necessary condition for a "flip-flop" transition. ${ }^{20,21}$ In fact, this poor contact with the electron spin-spin interaction reservoir at low temperatures makes it possible to cool the deuteron-quadrupole reservoir quite efficiently.

After reaching a common spin temperature, $\alpha$ and $\beta$ decay with a common rate $\tau_{\mathrm{rf}}^{-1}$, given by the weighted average of the proton-spin-lattice relaxation time $T_{1 H}$ and $T_{A_{D}},{ }^{7}$

$$
\begin{gathered}
\tau_{\mathrm{rf}}^{-1}=\left(\Delta^{2} T_{1 H}^{-1}+\Omega^{2} T_{A D}^{-1}\right) /\left(\Delta^{2}+\Omega^{2}\right), \\
\text { or since } T_{1 H} \gg T_{A_{D}}, \\
\tau_{\mathrm{rl}}=T_{A_{D}}\left(1+\Delta^{2} / \Omega^{2}\right) .
\end{gathered}
$$

\section{EXPERIMENTAL RESULTS}

The experiments were performed around $0.1 \mathrm{~K}$ in a ${ }^{3} \mathrm{He}-{ }^{4} \mathrm{He}$ dilution refrigerator, ${ }^{22}$ which was $10-$ cated in a 25-kG magnetic field. Dynamic polarization could be obtained by applying microwaves to a multimode cavity, containing a $1-2 \mathrm{~cm}^{3}$ sample, which was sealed into cylindrical bags of fluorinated ethylene propylene (FEP) foil. The polarization was obtained by comparing cw magnetic resonance signals of different nuclear species with one another after dynamic polarization or by comparing them with the ones measured in thermal equilibrium with the lattice at a known temperature. ${ }^{2}$ The NMR signals were detected by $Q$ meters, which used one single coil inside the cavity. This coil was tuned with capacitors in series to the resonant frequencies of the different nuclei (106.5 $\mathrm{MHz}$ for protons and 16.3 $\mathrm{MHz}$ for deuterons in a 25-kG magnetic field). A small computer was used to average the signals and to calculate the polariza- 
tions. The samples consisted of partially deuterated propanediol-D6 $\left[\mathrm{C}_{3} \mathrm{D}_{6}(\mathrm{OH})_{2}\right]$, in which paramagnetic $\mathrm{Cr}^{5+}$ complexes could be introduced via a chemical reaction with potassium dichromate ${ }^{23}$ by heating and stirring a mixture of $10 \mathrm{~cm}^{3}$ propanediol and $2 \mathrm{~g}$ of $\mathrm{K}_{2} \mathrm{Cr}_{2} \mathrm{O}_{7}$ at $70^{\circ} \mathrm{C}$ under vacuum ${ }^{24}$ until a $\mathrm{Cr}^{5+}$ concentration of about $5 \times 10^{19}$ spins/ $\mathrm{cm}^{3}$ was obtained after about $90 \mathrm{~min}$. Then the concentration was doubled by quickly distilling part of the propanediol out of the solution. ${ }^{25}$ The obtained concentration of about $10^{20}$ spins $/ \mathrm{cm}^{3}$, which can be checked by just measuring the line-
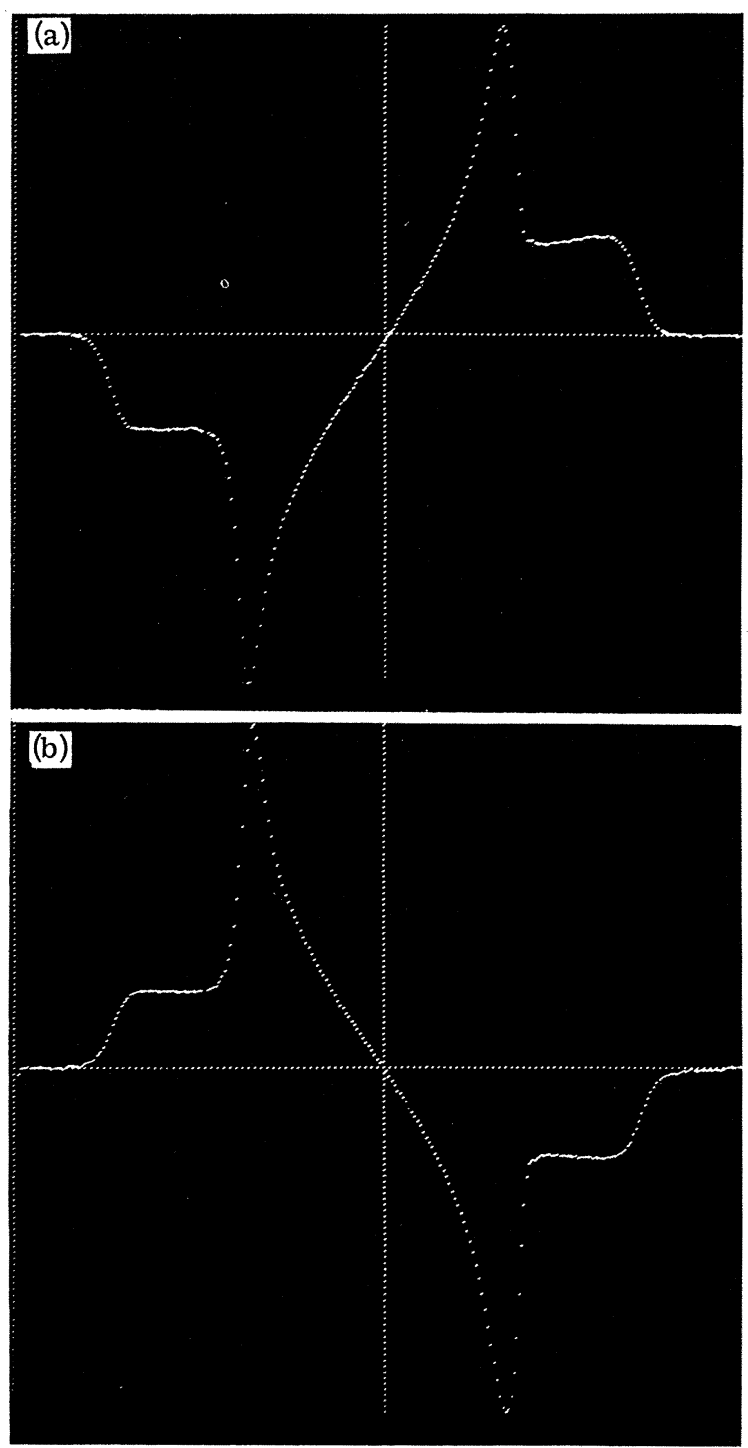

FIG. 2. (a) Deuteron signal after rf irradiation at 106. $640 \mathrm{MHz}$ of the proton-spin system with initial conditions: $P_{H}=-89 \%$ and $P_{D}=0 \%$. The peak alignment is $+25 \%$, the pedestal alignment is $-58 \%$. (b) Deuteron signal after rf irradiation at $106.410 \mathrm{MHz}$ with initial conditions $P_{H}=-88 \%$ and $P_{D}=0 \%$. The peak alignment is $-30 \%$; the pedestal alignment is $+48 \%$.

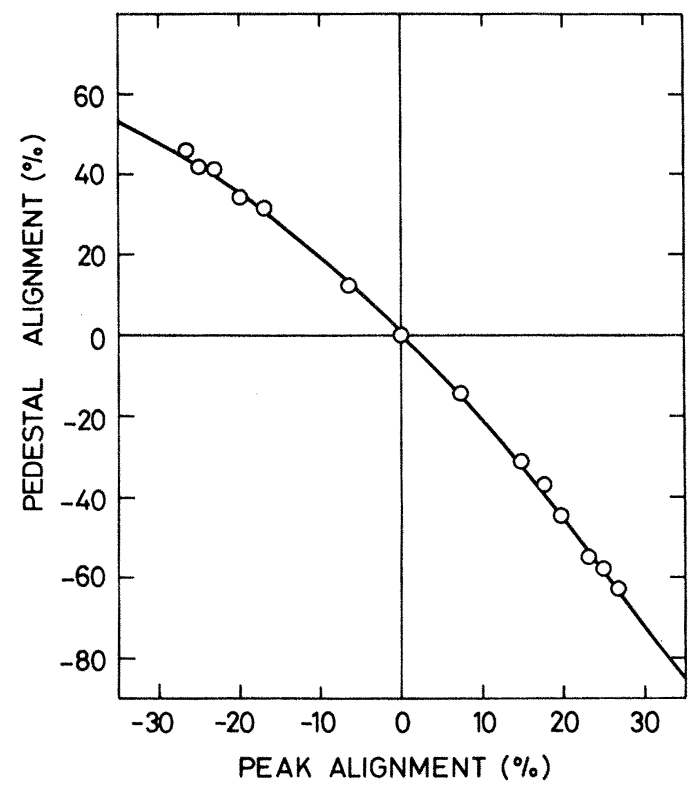

FIG. 3. Steady-state values of pedestal alignment vs peak alignment. The curve corresponds to equal quadrupole spin temperatures [deduced from Eq. (19)].

width of the ESR line, ${ }^{24,25}$ has been found to give maximum polarization. The $\mathrm{OH}$ groups in the partially deuterated propanediol appeared to be essential for the reaction and should not be replaced by OD groups. ${ }^{26}$

The experimental sequence is as follows: After dynamic polarization, the microwaves are switched off and the deuteron polarization is reduced to zero by a saturating rf field. Then the still highly polarized proton system is irradiated by a slightly off-resonance rf field, which causes the deuteronmagnetic-resonance signal to grow with an antisymmetrical shape, as shown in Fig. 2. The rf field strength needs to be only a few $m G$. We repeated this experiment for several values of the initial proton polarization and for different $\Delta$ 's.

During rf irradiation, the microwaves should be switched off, because they provide a strong thermal coupling between the electron spin-spin interaction reservoir and the nuclear Zeeman reservoirs. For example, with a microwave power of a few $\mathrm{mW} / \mathrm{g}$, all the nuclei obtain an equal spin temperature in a few seconds, independent of the lattice temperature. ${ }^{2}$ This means that a pure deuteron tensor polarization will also disappear in a few seconds, since it does not correspond to a homogeneous spin temperature within the deuteronspin system (the signals in Fig. 2 have positive and negative parts!).

The alignment is different for peaks and pedestals, because of their different values of $X$ $=\nu_{Q}\left(3 \cos ^{2} \theta-1\right)$. We denote them by $A_{\text {peak }}$ and $A_{\text {peds }}$ respectively. 


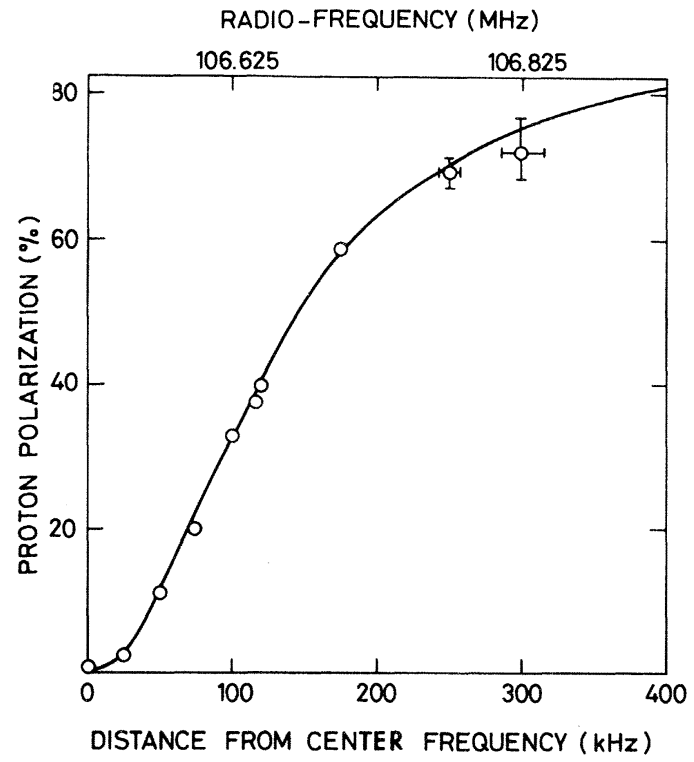

FIG. 4. Steady-state value of the final proton polarization under rf irradiation as a function of the radio frequency. The initial polarization was $90 \%$ each time. The curve was deduced from Eq. (17).

The alignment values can be determined from the deuteron-magnetic-resonance line, since $A_{\text {peak }}$ and $A_{\text {ped }}$ are proportional to the difference in intensity between the two peaks and between the outside parts of the pedestals, respectively, (see Sec. II B).

In Fig. 3 we plotted the values of $A_{\text {peak }}$ and $A_{\text {ped }}$ corresponding to the same spin temperature, to-

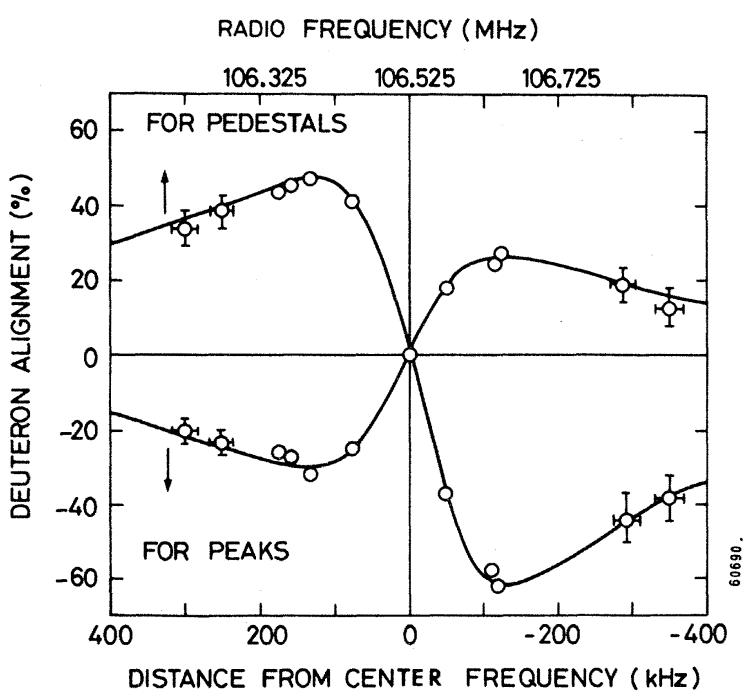

FIG. 5. Steady-state values of the alignment as function of the radio frequency. The initial proton polarization was $-90 \%$. The curves were deduced from Eqs. (16) and (19). The error bars are only shown when they exceed a relative error of $10 \%$.

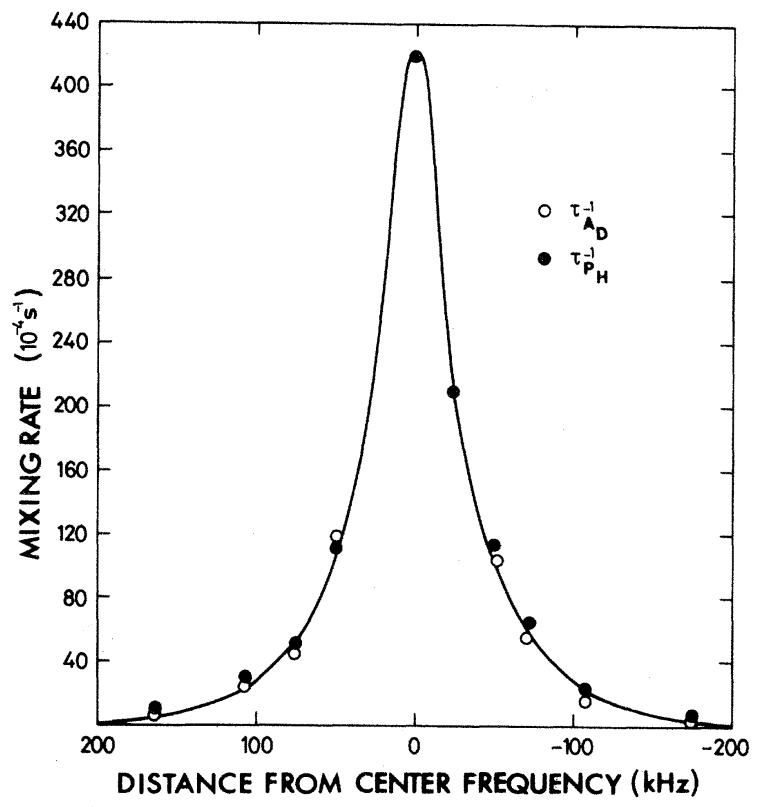

FIG. 6. Inverse of decay time of the proton polarization $(\bullet)$ and build-up time of the alignment (0) vs $\Delta$. The curve was calculated from Eq. (20), using the measured proton NMR line shape. The transition probability $W$ in Eq. (20) was determined by saturating the proton spin system at $\Delta=0$.

gether with the experimental values of the alignment in the steady state, which was taken as the time when $A_{\text {ped }}$ passed through its maximum; it decayed afterwards owing to finite spin-lattice re-

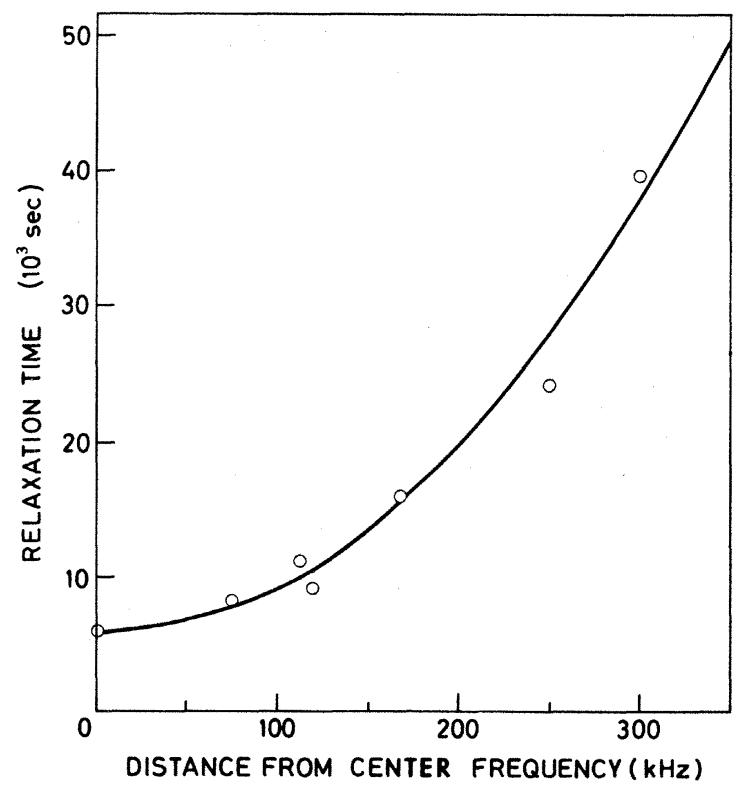

FIG. 7. Relaxation time of the common inverse spin temperature $\beta$ in the rotating frame as calculated from Eq. (22) together with the experimental points. 


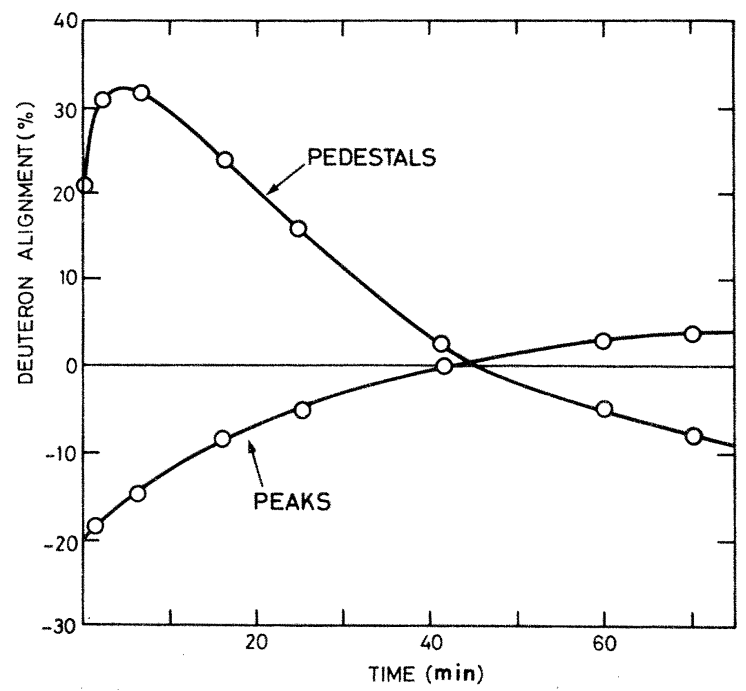

FIG. 8. Evolution of pedestal and peak alignment starting from an internal nonequilibrium. The pedestal alignment (upper curve) first increases instead of decreasing, which is attributed to the fact that at the chosen radio frequency, the $\mathrm{rf}$ field provides a strong thermal coupling between the pedestal deuterons and the remaining colder part of the quadrupole reservoir.

laxation. It can be seen that in the steady state, the quadrupole interaction reservoir can be described by one single spin temperature.

The experimental values of the final proton polarization are plotted in Fig. 4, together with a curve deduced from Eq. (17) with a value of $\Omega$

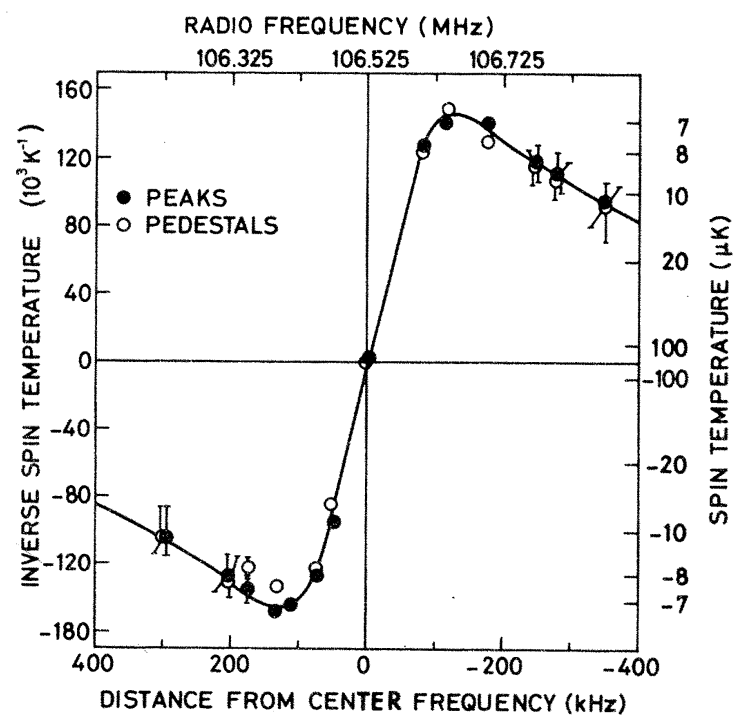

FIG. 9. Inverse spin temperature as a function of the radio frequency. The curve was calculated from Eq. (16). The initial proton polarization was $-90 \%$ each time. The points for $|\Delta|>150 \mathrm{kHz}$ were corrected for finite spinlattice relaxation, which caused a larger error. If the relative errors are less than $10 \%$, they are not indicated.

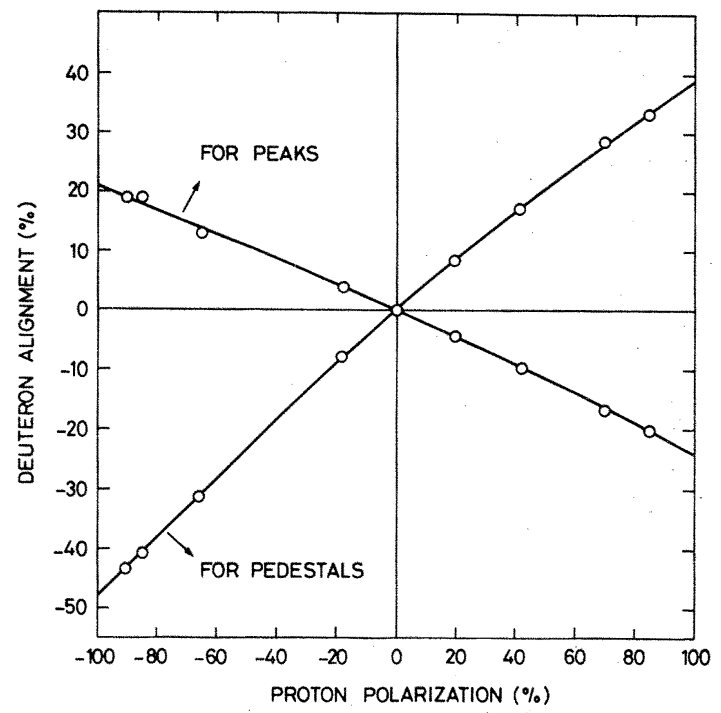

FIG. 10。 Final deuteron alignment as function of the initial proton polarization with a constant $\Delta$ equal to -80 $\mathrm{kHz}$. The curves were deduced from Eqs. (16) and (19). The relative errors are less than $10 \%$.

equal to $130 \mathrm{kHz}$. With this value, the best fit to the experimental points was obtained. This value of $\Omega$, which seems reasonable as compared to what can be expected from the NMR line widths, was also used for calculating the curves of the following figures, unless stated differently. The two highest points were corrected for spin-lattice relaxation.

Figure 5 shows the steady-state values of $A_{\text {peak }}$ and $A_{\text {ped }}$ as function of $\Delta$, which is the difference between proton Larmor frequency and radio frequency. The initial proton polarization was $-90 \%$. The curve was calculated with the aid of Eqs. (16) and (19). The points for $|\Delta|>150 \mathrm{kHz}$ were corrected for spin-lattice relaxation, because the alignment time strongly increased for larger $\Delta$, as can be seen from Fig. 6. Here we plotted the inverse of the time constants of the exponential development of $\alpha$ and $\beta$. The curve in this figure was calculated from Eq. (20), using for $h(\nu)$ an unsaturated experimental proton NMR line shape. The spin-lattice relaxation time of the common inverse spin temperature $\beta$ as function of $\Delta$ is shown in Fig. 7, together with the theoretical curve, deduced from Eq. (22).

The mixing rate was determined by neglecting the initial nonexponential variation of $P_{H}$ and $A_{D}$. The first nonexponential behavior is attributed to the following facts: (i) From the Provotorov equations, an exponential behavior is expected only if Eq. (6) is fulfilled; that is, if $P_{H} \lesssim 50 \%$, which is not true for the initial period; (ii) When $\Delta$ is much smaller than the total linewidth of the deuteron line $(230 \mathrm{kHz})$, the buildup of the alignment for the 

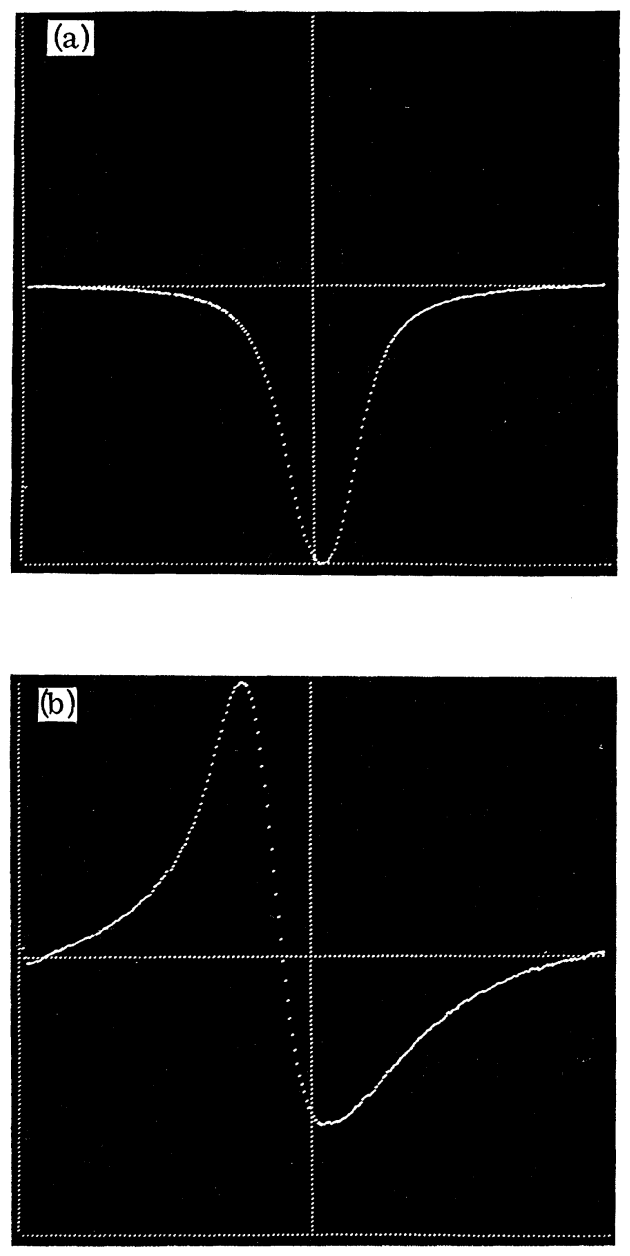

FIG. 11. (a) NMR signal of $-96 \%$ polarized protons in 1,2-propanediol. The frequency sweep on the horizontal axis is $400 \mathrm{kHz}$. (b) NMR signal of $-90 \%$ polarized protons after applying an intense $\mathrm{rf}$ field at the frequency where it crosses zero.

peaks is slightly shorter than for the pedestals in the beginning.

This may be due to the fact that the thermal contact for the pedestal deuterons is rather poor, owing to their low concentration, and they therefore cool down in a slightly longer time. The cross relaxation rate within the inhomogeneous deuteronspin system is rather slow, as was apparent from the fact that one can burn a "hole" in it by saturation of the DMR line at a fixed frequency. These holes recovered in about one minute, if they were located at the center, and in a slightly longer time, if they were located at the edges of the DMR line. ${ }^{2}$

The slightly different alignment times for peaks and pedestals in case of small values of $|\Delta|$ allows one to create a nonequilibrium within the quadrupole reservoir and to study the role of the forbidden transitions in establishing thermal equilibrium. The experiment can be done as follows: Starting from an initial proton polarization of $-91 \%$, an rf field with a frequency such that $\Delta=90$ $\mathrm{kHz}$ is applied during a few minutes, which results in $A_{\text {peak }}=-20 \%$ and $A_{\text {ped }}=+20 \%$; this implies that the peak deuterons are colder than the pedestal deuterons. The radio frequency is then changed so that $\Delta=-255 \mathrm{kHz} ;|\Delta|$ is then slightly larger than the total deuteron line span. The evolution of the alignment is then as shown in Fig. 8. Instead of decreasing, $A_{\text {ped }}$ first increases rapidly until the pedestal deuterons reach thermal equilibrium with the remaining part of the quadrupole reservoir. Then $A_{\text {peak }}$ and $A_{\text {ped }}$ start to evolve towards their equilibrium value, which is of an opposite sign, since $\Delta$ has changed sign. The rapid initial increase of $A_{\text {ped }}$ may be due to the fact that at this particular radio frequency, the rf field provides a strong thermal contact for the pedestal deuterons via the forbidden transitions in which one proton and two pedestal deuterons flip simultaneously. Similar thermal mixing experiments have been performed with nuclei in thermal contact with an electron spin-spin interaction reservoir. ${ }^{2}$

The steady-state values of $\beta$ are plotted as a function of the radio frequency in Fig. 9. The peak values of $\beta$ correspond to quadrupole temperatures of $\pm(7 \pm 0.7) \mu \mathrm{K}$.

Figure 10 shows the deuteron alignment as a function of the initial proton polarization; during each measurement $\Delta$ was $-80 \mathrm{kHz}$. These measurements were done in ethanediol-D4, ${ }^{16}$ and in order to calculate the curves [from Eqs. (16) and (19)], we used slightly different values of $\Omega$ and $\nu_{Q}$, these being 140 and $+18.5 \mathrm{kHz}$, respectively. The value of $\Omega$ was again chosen to give the best fit to the experimental points.

The influence of the proton spin-spin interaction reservoir was also evident from the change in the proton NMR signal during irradiation. It became zero at the irradiation frequency, but sometimes negative below this frequency and positive above, or the opposite way around according to the case. Such behavior was expected for homogeneous spin systems under strong saturation conditions, ${ }^{4,12}$ because the absorption signal is not proportional to the factor $\alpha \nu_{H}$, but

$$
h(\nu) \propto\left[\alpha \nu_{H}-\beta\left(\nu_{H}-\nu\right)\right],
$$

as follows from the provotorov equations.

If the spin system is in thermal equilibrium with the lattice, the second term can be neglected compared with the first one. However, during irradiation the second term may become very large and dependent on the sign of $\Delta$; it may be of an opposite sign compared with the first one, thus changing 
the sign of $h(\nu)$. Figure 11(b) shows the proton NMR signal after irradiation at the frequency where the signal crosses zero. It was initially $-90 \%$ polarized.

\section{DISCUSSION}

The fact that the inhomogeneous deuteron quad rupole reservoir can be described by one single spin-temperature makes it possible to apply the spin-temperature theory, although the Zeeman energy reservoir cannot be described by a spin temperature at all (the aligned DMR signal has positive and negative parts!). This latter fact clearly shows that the energies of the two reservoirs are separately constants of motion in a high-magnetic field, as they should because their operators commute with each other.

In the high-temperature approximation, one has the simple relation

$$
A_{\text {ped }}=-2 A_{\text {peak }}=-4 \beta \nu_{0},
$$

as follows from a series expansion of Eq. (19). At lower temperatures, where terms of higher order in $\beta$ are no longer negligible, deviations of this relation occur, as is apparent from Fig. 3 where we plotted $A_{\text {pod }}$ versus $A_{\text {poak }}$. The deviations are, however, $10 \%$ or smaller. In calculating the alignment from Eq. (19) for a given value of $\beta$, we did not neglect higher-order terms, although they were neglected in Eqs. (12) and (15), from which $\beta$ was calculated. The fact that the agreement between $\beta$ and the experimental data is still better than $10 \%$, is probably due to the fact that we used the heat capacity of the deuteron quadrupole reservoir as an adjustable parameter to fit the data.

Taking into account that the value of this parameter is reasonable as compared to what can be expected from the linewidths of the NMR lines, and the fact that its value can be used to describe a wide variety of data, I think we may conclude that these experiments provide satisfactory agreement with the following aspects of the spin-temperature theory: (a) cooling of a spin-spin interaction reservoir by slightly off-resonance irradiation of a spin system; (b) thermal mixing between subsystems of different nuclear-spin species in a highmagnetic field; (c) saturation of a nuclear-spin system as function of the irradiation rf; (d) spinlattice relaxation of a spin system during off-resonance irradiation.

Cooling of a quadrupole reservoir has been observed at much lower magnetic-field values in paradichlorobenzene, which was irradiated near the chlorine quadrupole-resonance-frequency. ${ }^{27}$ The cooling was apparent from the observed proton polarization. This experiment can be compared with ours in the respect that there a spinspin order produced polarization (or Zeeman order), while we first polarized and then transformed the Zeeman order into spin-spin order. However, our experiment provided semiquantitative results in contrast to the above-mentioned experiments where only qualitative features were observed. Furthermore, by dynamic polarization, we could choose the initial spin temperatures in a wide range, positive as well as negative.

\section{ACKNOWLEDGMENTS}

I wish to express my sincere thanks to the members of the Polarized Target Group at CERN for their help and interest during the course of this work. In particular, I wish to thank Dr. M. Borghini and Dr. K. Morimoto for many fruitful discussions. Thanks are also due to $\mathrm{H}$. Ponssen and Dr. F. Udo for constructing the sensitive NMR equipment and Dr. T. O. Niinikoski for designing the excellent working dilution refrigerator. Furthermore, I am grateful to Professor B. S. Blaisse, Dr. D. A. Hill, and to two of the abovementioned colleagues (M.B. and T.O.N.) for valuable comments on the manuscript.
*Present address: University of Michigan, Randall Laboratory of Physics, Ann Arbor, Mich. 48104.

${ }^{1} \mathrm{~W}$. de Boer and T. O. Niinikoski, Nucl. Instrum. Methods 114, 495 (1974); D. A. Hill (private communication).

${ }^{2}$ W. de Boer, M. Borghini, K. Morimoto, T. O. Niinikoski, and F. Udo, J. Low Temp. Phys. 15, 249 (1974).

${ }^{3} \mathrm{~W}$. de Boer, thesis (Delft University of Technology, Delft, (1974).

${ }^{4}$ B. N. Provotorov, Zh. Eksp. Teor. Fiz. 41, 1582 (1961) [Sov. Phys.-JETP 14, 1126 (1962)]; Fiz. Tver. Tela 4 , 2940 (1962) [Sov. Phys. Solid State $\underline{4}, 2155$ (1963)]; Phys. Rev. 128, 75 (1962).

${ }^{5}$ A. G. Redfield, Phys. Rev. 98, 1787 (1955).

${ }^{6} \mathrm{~A}$. Abragam, Principles of Nuclear Magnetism (Oxford U. P., Lond, 1961).
${ }^{7} \mathrm{M}$. Goldman, Spin Temperature and Magnetic Resonance in Solids (Oxford U. P., London, 1970), and references quoted therein.

${ }^{8}$ A. G. Anderson and S. R. Hartmann, Phys. Rev. 120, 2023 (1962).

${ }^{9}$ J. Philippot, Phys. Rev. 133, A471 (1964).

${ }^{10} \mathrm{~J}$. Jeener, in Advances in Magnetic Resonance, edited by J. S. Waugh (Academic, New York, 1968), Vol. 3, pp. 206-320. J. Jeener, H. Eisendraht, and K. van Steenwinkel, Phys. Rev. A 133, 478 (1964).

${ }^{11}$ L. C. Hebel, Solid State Physics edited by F. Seitz and D. Turnbull (Academic, New York, 1963), Vol. 15, p. 409.

${ }^{12}$ V. A. Atsarkin and M. I. Rodak, Usp. Fiz. Nauk 107, 3 (1972) [Sov. Phys. Usp. 15, 251 (1973)], and references quoted therein. 
${ }^{13}$ W。Th. Wenckebach and N. J. Poulis, Seventeen Congress Ampère, Turku, Finland, 1972, edited by V. Hovi (North-Holland, Amsterdam, 1973), p. 120, and references quoted therein.

${ }^{14} \mathrm{M}$. Borghini, Proceedings of the Second International Conference on Polarized Targets, Berkeley, 1971, edited by G. Shapiro, (LBL 500, UC-34 Physics, National Technical Information Service, Springfield, Va., 1972), p. 1, and references quoted therein.

${ }^{15}$ M. E. Zhabotinskii, A。E. Mefed, and M. I. Rodak, Zh. Eksp. Teor. Fiz. 61, 1917 (1971) [Sov. Phys.-JETP 34,1020 (1972)].

${ }^{16} \overline{\mathrm{W}}$. de Boer, M. Borghini, K. Morimoto, T。O. Niinikoski, and F. Udo, Phys. Lett. A 46, 143 (1973).

${ }^{17}$ R. J. Blin-Stoyle and M. A. Grace, Handb. Phys. 42, 555 (1957)

${ }^{18}$ E. L. Lucken, Nuclear Quadrupole Coupling Constants
(Academic, London, 1969), and references quoted therein.

${ }^{19}$ N. Bloembergen, Physica (Utr.) 15, 386 (1949).

${ }^{20} \mathrm{~T}$. E. Gunther and C. D. Jeffries, Phys. Rev. 159, 290 (1967)

${ }^{21}$ S. F. J. Cox, V. Bouffard, and M. Goldman, J. Phys. C 7, L100 (1973).

${ }^{22}$ T. O. Ninikoski, Nucl. Instrum. Methods 97,95 (1971).

${ }^{23}$ N. S. Garif'yanov, B. M. Kozyrev, and V. N. Fedotov, Dokl. Akad. Nauk SSSR 178, 808 (1968) [Sov. Phys. Dokl. 13, 107 (1968)].

${ }^{24} \mathrm{H}$. Glättli, in Ref. 14, p. 281.

${ }^{25}$ W. de Boer, Nucl. Instrum. Methods 107, 99 (1973). ${ }^{26}$ This product can be obtained from: Merck, Sharp and Dohme Ltd., P. O. Box 899, Quebec, Canada.

${ }^{27} \mathrm{M}$. Goldman and A. Landesman, Phys. Rev。132, 610 (1963). 